\title{
ON THE MOTION OF A BODY IN THERMAL EQUILIBRIUM IMMERSED IN A PERFECT GAS*
}

\author{
Kazuo Aoki $^{1}$, Guido Cavallaro ${ }^{2}$, Carlo Marchioro ${ }^{2}$ and Mario Pulvirenti ${ }^{2}$
}

\begin{abstract}
We consider a body immersed in a perfect gas and moving under the action of a constant force. Body and gas are in thermal equilibrium. We assume a stochastic interaction body/medium: when a particle of the medium hits the body, it is absorbed and immediately re-emitted with a Maxwellian distribution. This system gives rise to a microscopic model of friction. We study the approach of the body velocity $V(t)$ to the limiting velocity $V_{\infty}$ and prove that, under suitable small-
\end{abstract} ness assumptions, the approach to equilibrium is

$$
\left|V(t)-V_{\infty}\right| \approx \frac{C}{t^{d+1}}
$$

where $d$ is the dimension of the space, and $C$ is a positive constant. This approach is not exponential, as typical in friction problems, and even slower than for the same problem with elastic collisions.

Mathematics Subject Classification. 76P05, 82B40, 82C40, 35L45, 35L50.

Received March 31, 2007. Revised October 16, 2007.

\section{INTRODUCTION}

We consider a body moving under the action of a constant force and immersed in a perfect gas, namely we assume that the one-particle distribution function of the gas evolves according to the free transport equation. The gas is initially at thermal equilibrium. The boundary conditions on the surface of the body are assumed of diffusive type: when a particle of the medium hits the body, it is absorbed and immediately re-emitted with a Maxwellian distribution at the same temperature of the gas.

This system represents a microscopic model of friction, for which we want to study the approach to equilibrium of the velocity of the body $V(t)$. In a friction problem we may expect an exponential approach to the limiting velocity $V_{\infty}$, but in the present case it is not so: due to the recollisions the approach turns out to obey the following power law

$$
\left|V(t)-V_{\infty}\right| \approx \frac{C}{t^{d+1}}
$$

\footnotetext{
Keywords and phrases. Kinetic theory of gases, Boltzmann equation, free molecular gas, friction problem, approach to equilibrium.

* Work performed under the auspices of GNFM-INDAM and the Italian Ministry of the University (MIUR).

1 Department of Mechanical Engineering and Science and Advanced Research Institute of Fluid Science and Engineering, Graduate School of Engineering, Kyoto University, Kyoto 606-8501, Japan. aoki@aero.mbox.media.kyoto-u.ac.jp

2 Dipartimento di Matematica, Università di Roma "La Sapienza", Piazzale A. Moro 2, 00185, Roma, Italy.

cavallar@mat.uniroma1.it; marchior@mat.uniroma1.it; pulvirenti@mat.uniroma1.it
} 
even slower than the law appearing in the elastic case (which states the time behavior $t^{-d-2}$ ), investigated in recent papers [3,4]. This result will be proved assuming the initial velocity $V_{0}$ sufficiently large and close enough to $V_{\infty}$. This asymptotics, rather surprising, depends on the fact that the time interval between two collisions of a gas particle with the body may be very large, so that the process has a very long tail memory. We note that in the present case the number of recollisions is larger compared with the elastic case studied in references [3,4]. This feature is responsible for the different time decay (1.1).

We can ask whether it is likely that a particle of the medium hits twice the body. It depends on the velocity of the obstacle, which must be comparable with a typical velocity of the medium (of the order of the sound speed). This can happen either for low temperatures or for particular physical situations.

Finally we remark that the long memory effect of the present model depends on the deterministic motion of the fluid particles. When we add a small stochasticity in the background, for instance due to the presence of small impurities, we expect that the power law approach remains valid for a long time, after which the exponential law holds asymptotically, since the long tail effects of the recollisions should be destroyed by the stochasticity.

The plan of the paper is the following: in Section 2 we introduce the model and state the results, while the proofs are given in Section 3.

\section{Model AND RESUlts}

The body we consider is a cylinder in $\mathbb{R}^{3}$, with circular basis of radius $R$ orthogonal to the $x$-axis and centered on the same axis, and height equal to $L$. We take for simplicity the cylinder with unitary mass and constant density. By the symmetry of the problem we are going to consider, it will be evident that the cylinder cannot undergo any kind of rotation, if initially absent (as we suppose), and its center of mass can move along the $x$-axis only. We denote by $X(t)$ the position of the center of mass at time $t$.

The body is immersed in a perfect gas in equilibrium at inverse temperature proportional to $\beta$ and with constant density $\rho$. The body interacts with the gas by means of boundary conditions on the one-particle distribution function which will be established later on. In addition the body is subject to a constant force $E$ directed along the $x$-axis.

We are interested in the time asymptotics of the system, in particular we want to establish whether and how the body reaches a limiting velocity.

We assume the perfect gas in the mean-field approximation. This means that we are considering the continuum limit for the free gas (see the Appendix in [3]). The presence of the body modifies the equilibrium of the gas, which starts to evolve according to the free transport equation. Let $f(x, v ; t),(x, v) \in \mathbb{R}^{3} \times \mathbb{R}^{3}$, be the mass density in the phase space of the gas particles, then

$$
\left(\partial_{t}+v \cdot \nabla_{x}\right) f(x, v ; t)=0, \quad \text { for } x \notin \bar{\Omega}_{t} .
$$

Here $\Omega_{t}$ denotes the domain of $\mathbb{R}^{3}$ occupied by the cylinder at time $t$.

We define

$$
f_{ \pm}(x, v ; t)=\lim _{\varepsilon \rightarrow 0^{+}} f(x \pm \varepsilon v, v ; t \pm \varepsilon),
$$

and assume as initial state for the gas distribution the thermal equilibrium, namely

$$
\begin{array}{ll}
f_{+}(x, v ; 0)=\rho\left(\frac{\beta}{\pi}\right)^{3 / 2} \mathrm{e}^{-\beta v^{2}}, & \text { for } x \notin \Omega_{0}, \\
f_{+}(x, v ; 0)=0, & \text { for } x \in \Omega_{0},
\end{array}
$$

for $\beta>0$.

On the boundary of the cylinder at time $t$, i.e. on $\partial \Omega_{t}$, we impose diffusive boundary conditions: a gas particle is first absorbed, and then re-emitted with the equilibrium distribution. We treat separately the right basis $\left(\partial \Omega_{t}^{+}\right)$, the left basis $\left(\partial \Omega_{t}^{-}\right)$, and the lateral surface $\left(\partial \Omega_{t}^{\ell}\right)$. Denoting by $v_{x}$ and $v_{\perp}$ the velocity components 
of the gas particles on the $x$-axis and on the orthogonal plane respectively, we impose that on $\partial \Omega_{t}^{+}$the gas particles are emitted according to the distribution

$$
f_{+}(x, v ; t)=\alpha J(x, t) \mathrm{e}^{-\beta\left(v_{x}-V(t)\right)^{2}} \mathrm{e}^{-\beta v_{\perp}^{2}}, \quad x \in \partial \Omega_{t}^{+},
$$

with $v_{x} \geq V(t)$ (the particles are emitted outside the body). The constant $\alpha$ and the function $J(x, t)$ are determined imposing that the number of particles absorbed per unit time and per unit surface by $\partial \Omega_{t}^{+}$is equal to the number of particles re-emitted per unit time and per unit surface by $\partial \Omega_{t}^{+}$, due to mass conservation. Therefore

$$
\begin{gathered}
\int_{v_{x} \leq V(t)} \mathrm{d} v\left(V(t)-v_{x}\right) f_{-}(x, v ; t)= \\
\alpha J(x, t) \int \mathrm{d} v_{\perp} \mathrm{e}^{-\beta v_{\perp}^{2}} \int_{v_{x} \geq V(t)} \mathrm{d} v_{x}\left(v_{x}-V(t)\right) \mathrm{e}^{-\beta\left(v_{x}-V(t)\right)^{2}},
\end{gathered}
$$

so we can identify $J(x, t)$ with the ingoing flux,

$$
J(x, t)=\int_{v_{x} \leq V(t)} \mathrm{d} v\left(V(t)-v_{x}\right) f_{-}(x, v ; t), \quad x \in \partial \Omega_{t}^{+},
$$

choosing

$$
\alpha=\left[\left(\int_{-\infty}^{+\infty} \mathrm{e}^{-\beta x^{2}} \mathrm{~d} x\right)^{2} \int_{0}^{+\infty} x \mathrm{e}^{-\beta x^{2}} \mathrm{~d} x\right]^{-1} .
$$

On the left basis of the cylinder we can repeat the same considerations, the only modification consisting in the definition of the flux which, for $x \in \partial \Omega_{t}^{-}$, is:

$$
J(x, t)=\int_{v_{x} \geq V(t)} \mathrm{d} v\left(v_{x}-V(t)\right) f_{-}(x, v ; t) .
$$

On the lateral surface we denote by $\hat{n}$ the outward unit normal, calculated at the absorption (or emission) point of the gas particle. The flux balance yields:

$$
\begin{gathered}
\int \mathrm{d} v \chi\left(\left\{v_{\perp} \cdot \hat{n} \leq 0\right\}\right)\left|v_{\perp} \cdot \hat{n}\right| f_{-}(x, v ; t)= \\
\bar{\alpha} J(x, t) \int \mathrm{d} v \chi\left(\left\{v_{\perp} \cdot \hat{n} \geq 0\right\}\right)\left|v_{\perp} \cdot \hat{n}\right| \mathrm{e}^{-\beta\left(v_{x}-V(t)\right)^{2}} \mathrm{e}^{-\beta v_{\perp}^{2}},
\end{gathered}
$$

then choosing

$$
\bar{\alpha}=\left[\int_{-\infty}^{+\infty} \mathrm{d} v_{x} \mathrm{e}^{-\beta\left(v_{x}-V(t)\right)^{2}} \int \mathrm{d} v_{\perp} \chi\left(\left\{v_{\perp} \cdot \hat{n} \geq 0\right\}\right)\left|v_{\perp} \cdot \hat{n}\right| \mathrm{e}^{-\beta v_{\perp}^{2}}\right]^{-1},
$$

(notice that it results $\bar{\alpha}=\alpha$ ) we have, for $x \in \partial \Omega_{t}^{\ell}$,

$$
J(x, t)=\int \mathrm{d} v \chi\left(\left\{v_{\perp} \cdot \hat{n} \leq 0\right\}\right)\left|v_{\perp} \cdot \hat{n}\right| f_{-}(x, v ; t) .
$$

Equation (2.1) is complemented by the evolution equation for the body:

$$
\begin{gathered}
\dot{X}(t)=V(t), \quad \dot{V}(t)=E-F(t), \\
X(0)=0, \quad V(0)=V_{0},
\end{gathered}
$$


where $E>0$ is a constant given field and $F(t)$ is the action of the gas on the body:

$$
\begin{aligned}
F(t)= & \int_{\partial \Omega_{t}^{+}} \mathrm{d} x \int_{v_{x} \leq V(t)} \mathrm{d} v\left(V(t)-v_{x}\right)^{2} f_{-}(x, v ; t) \\
& -\int_{\partial \Omega_{t}^{-}} \mathrm{d} x \int_{v_{x} \geq V(t)} \mathrm{d} v\left(V(t)-v_{x}\right)^{2} f_{-}(x, v ; t) \\
& +\int_{\partial \Omega_{t}^{+}} \mathrm{d} x \int_{v_{x} \geq V(t)} \mathrm{d} v\left(v_{x}-V(t)\right)^{2} f_{+}(x, v ; t) \\
& -\int_{\partial \Omega_{t}^{-}} \mathrm{d} x \int_{v_{x} \leq V(t)} \mathrm{d} v\left(v_{x}-V(t)\right)^{2} f_{+}(x, v ; t) \\
& +\int_{\partial \Omega_{t}^{\ell}} \mathrm{d} \sigma \int_{v_{x} \leq V(t)} \mathrm{d} v \chi_{\perp}^{-}\left|v_{\perp} \cdot \hat{n}\right|\left|V(t)-v_{x}\right| f_{-}(x, v ; t) \\
& -\int_{\partial \Omega_{t}^{\ell}} \mathrm{d} \sigma \int_{v_{x} \geq V(t)} \mathrm{d} v \chi_{\perp}^{-}\left|v_{\perp} \cdot \hat{n}\right|\left|V(t)-v_{x}\right| f_{-}(x, v ; t) \\
& +\int_{\partial \Omega_{t}^{\ell}} \mathrm{d} \sigma \int_{v_{x} \geq V(t)} \mathrm{d} v \chi_{\perp}^{+}\left|v_{\perp} \cdot \hat{n}\right|\left|V(t)-v_{x}\right| f_{+}(x, v ; t) \\
& -\int_{\partial \Omega_{t}^{\ell}} \mathrm{d} \sigma \int_{v_{x} \leq V(t)} \mathrm{d} v \chi_{\perp}^{+}\left|v_{\perp} \cdot \hat{n}\right|\left|V(t)-v_{x}\right| f_{+}(x, v ; t),
\end{aligned}
$$

where $\mathrm{d} \sigma$ is the surface element on $\partial \Omega_{t}^{\ell}$, and we put $\chi_{\perp}^{+}=\chi\left(\left\{v_{\perp} \cdot \hat{n} \geq 0\right\}\right), \chi_{\perp}^{-}=\chi\left(\left\{v_{\perp} \cdot \hat{n} \leq 0\right\}\right)$. The first and the second lines of (2.11) take into account the momentum transfer from gas particles absorbed by $\partial \Omega_{t}^{+}$ and $\partial \Omega_{t}^{-}$respectively, the third and the fourth lines the momentum transfer from particles emitted by $\partial \Omega_{t}^{+}$ and $\partial \Omega_{t}^{-}$, the fifth and the sixth lines the momentum transfer from particles absorbed by $\partial \Omega_{t}^{\ell}$, and finally the seventh and the eighth lines, which turn out to cancel each other, the momentum transfer from particles emitted by $\partial \Omega_{t}^{\ell}$. For a heuristic derivation of this formula in the elastic case see [3].

A similar model with elastic collisions between fluid particles and body has been introduced in connection with the so called piston problem (see $[7,8]$ and references quoted therein). An investigation on the approach to equilibrium in such a model has been done in [3,4]. A comparison between the results obtained in these references and the results given in the present paper will be done just after the statement of Theorem 2.1.

A few words to explain the reason why we assume the mean field limit. If we consider the medium as a gas of light particles with small but finite mass, the collisions between particles and body give rise to a very irregular motion. However the averaged motion of the body is expected to be regular when the ratio between the mass of the gas particles and that of the body vanishes. To avoid the difficulties connected with the computation of averaged quantities we assume the mean field limit, namely the number of particles per unit volume diverges while their mass vanishes, in such a way that the density stays bounded. This limit is well known for interacting particle systems (see $[1,2,6,9,10]$ ).

Summarizing we define a solution of our problem to be any pair $(f, V)$ where $V=V(t)$ solves, for almost all $t \in \mathbb{R}^{+}$, equations (2.10), (2.11) and $f$ satisfies equation (2.12) below

$$
\frac{\mathrm{d}}{\mathrm{d} t} f(x+v t, v ; t)=0 \quad \text { a.e. }(x, v),
$$

together with the previously described conditions on $\partial \Omega_{t}$, and the initial condition (2.3). The existence of this solution can be proved by compactness (see later on), while the uniqueness has not been investigated. 
Indeed while the asymptotic behavior can be outlined by analyzing few recollisions, in order to get uniqueness we need a complete control of the full collision sequence,which is beyond the techniques of the present paper.

Notice that, assuming the time evolution of the body is given, we could follow back in time the trajectory of a gas particle with position $x$ and velocity $v$ at time $t$. For a particle hitting the body at time $t$, we denote by $\tau$ the recollision time (in the past). If $\tau$ does not exist, we say that the particle has no recollisions.

Notice that, for obvious geometrical reasons, particles hitting the body on $\partial \Omega_{t}^{\ell}$ at time $t$ have no recollisions. Hence for $f_{-}(x, v ; t)$ in $(2.9)$ and $(2.11)$, on $\partial \Omega_{t}^{\ell}$, we have to put the initial distribution $(2.3)$.

It is convenient to rewrite the term $F(t)$ in (2.11) in a more compact form, by expressing $f_{+}$in terms of $f_{-}$by using (2.4), (2.6), (2.7) and (2.9):

$$
\begin{aligned}
F(t)= & \int_{\partial \Omega_{t}^{+}} \mathrm{d} x \int_{v_{x} \leq V(t)} \mathrm{d} v\left[\left(V(t)-v_{x}\right)^{2}+\lambda\left|V(t)-v_{x}\right|\right] f_{-}(x, v ; t) \\
& -\int_{\partial \Omega_{t}^{-}} \mathrm{d} x \int_{v_{x} \geq V(t)} \mathrm{d} v\left[\left(V(t)-v_{x}\right)^{2}+\lambda\left|V(t)-v_{x}\right|\right] f_{-}(x, v ; t) \\
& +k \int_{-\infty}^{V(t)} \mathrm{d} v_{x}\left|V(t)-v_{x}\right| \mathrm{e}^{-\beta v_{x}^{2}}-k \int_{V(t)}^{+\infty} \mathrm{d} v_{x}\left|V(t)-v_{x}\right| \mathrm{e}^{-\beta v_{x}^{2}},
\end{aligned}
$$

where

and

$$
\lambda=\alpha \int \mathrm{d} v_{\perp} \mathrm{e}^{-\beta v_{\perp}^{2}} \int_{0}^{+\infty} \mathrm{d} x x^{2} \mathrm{e}^{-\beta x^{2}},
$$

$$
k=\rho\left(\frac{\beta}{\pi}\right)^{\frac{3}{2}} \int_{\partial \Omega_{t}^{\ell}} \mathrm{d} \sigma \int \mathrm{d} v_{\perp} \chi_{\perp}^{-}\left|v_{\perp} \cdot \hat{n}\right| \mathrm{e}^{-\beta v_{\perp}^{2}} .
$$

Neglecting the recollisions between the gas particles and the body, the friction term is easily computed, putting in place of $f_{-}(x, v ; t)$ the initial distribution $(2.3)$. This friction term is:

$$
\begin{aligned}
F_{0}(V)= & \bar{C} \int_{-\infty}^{V(t)} \mathrm{d} v_{x}\left[\left(V(t)-v_{x}\right)^{2}+\bar{\lambda}\left|V(t)-v_{x}\right|\right] \mathrm{e}^{-\beta v_{x}^{2}} \\
& -\bar{C} \int_{V(t)}^{+\infty} \mathrm{d} v_{x}\left[\left(V(t)-v_{x}\right)^{2}+\bar{\lambda}\left|V(t)-v_{x}\right|\right] \mathrm{e}^{-\beta v_{x}^{2}}
\end{aligned}
$$

where

$$
\bar{C}=\rho\left(\frac{\beta}{\pi}\right)^{\frac{3}{2}} \int_{\partial \Omega_{t}^{+}} \mathrm{d} x \int \mathrm{d} v_{\perp} \mathrm{e}^{-\beta v_{\perp}^{2}}, \quad \bar{\lambda}=\lambda+k / \bar{C} .
$$

We remark that, proceeding as in reference [3], it can be easily seen that $F_{0}$ is a positive, increasing and convex function in the interval $(0,+\infty)$. Therefore there exists a unique solution $V_{\infty}$ of $F_{0}\left(V_{\infty}\right)=E$. We assume that $V_{\infty} \geq V_{0}>0$.

Let us show that, without recollisions, our problem can be trivially solved. Indeed replacing $F$ by $F_{0}$ in equation (2.10) we have:

$$
\begin{gathered}
\dot{X}(t)=V(t), \quad \dot{V}(t)=E-F_{0}(V(t))=K(t)\left(V_{\infty}-V(t)\right), \\
X(0)=0, \quad V(0)=V_{0},
\end{gathered}
$$


where

$$
K(t)=\frac{F_{0}\left(V_{\infty}\right)-F_{0}(V(t))}{V_{\infty}-V(t)}
$$

The solution to equation (2.15) can be almost explicitly computed. We can see that $V$ is increasing in time and converging to $V_{\infty}$, moreover a standard comparison argument shows that

$$
\gamma \mathrm{e}^{-C_{-} t} \leq V_{\infty}-V(t) \leq \gamma \mathrm{e}^{-C_{+} t}
$$

where

$$
\gamma=V_{\infty}-V_{0} \quad \text { and } \quad C_{+}=F_{0}^{\prime}\left(V_{0}\right) \leq C_{-}=F_{0}^{\prime}\left(V_{\infty}\right)
$$

The full problem, where we include recollisions, is much more difficult. Let us rewrite the full friction term $F$ as:

where $r^{+}(t)$ and $r^{-}(t)$ are:

$$
F(t)=F_{0}(V(t))+r^{+}(t)+r^{-}(t)
$$

$$
\begin{aligned}
& r^{+}(t)=\int_{\partial \Omega_{t}^{+}} \mathrm{d} x \int_{v_{x} \leq V(t)} \mathrm{d} v d\left(v_{x}, V(t)\right)\left[f_{-}(x, v ; t)-\rho\left(\frac{\beta}{\pi}\right)^{3 / 2} \mathrm{e}^{-\beta v^{2}}\right], \\
& r^{-}(t)=\int_{\partial \Omega_{t}^{-}} \mathrm{d} x \int_{v_{x} \geq V(t)} \mathrm{d} v d\left(v_{x}, V(t)\right)\left[\rho\left(\frac{\beta}{\pi}\right)^{3 / 2} \mathrm{e}^{-\beta v^{2}}-f_{-}(x, v ; t)\right],
\end{aligned}
$$

and

$$
d\left(v_{x}, V(t)\right)=\left(V(t)-v_{x}\right)^{2}+\lambda\left|V(t)-v_{x}\right|
$$

The quantities $\rho, \beta, R, L, V_{\infty}$ (or equivalently $E$ ), and $\gamma=V_{\infty}-V_{0}$ are the data of the problem.

We are now in the position to state the main result of the present paper. We give the result depending on the dimension $d=1,2,3$ of the physical space, treating explicitly the case $d=3$ (the remaining cases follow with obvious modifications).

Theorem 2.1. Let $(V(t), f(t))$ be a solution to problem $(2.1)-(2.11)$. Then there exist $\gamma_{0}=\gamma_{0}\left(\rho, \beta, R, L, V_{\infty}\right)>$ 0 sufficiently small and positive constants $C_{0}, A_{+}, A_{-}$independent of $\gamma$, such that, for any $\gamma \in\left(0, \gamma_{0}\right)$ and $V_{0}>C_{0}$, the solution $(V(t), f(t))$ satisfies the following properties:

(i) For any $t \geq 0$ :

$$
V_{\infty}-V(t) \leq \gamma \mathrm{e}^{-C_{+} t}+\gamma^{2} \frac{A_{+}}{(1+t)^{d+1}}
$$

(ii) There exists a sufficiently large $\bar{t}$, depending on $\gamma$, such that for any $t \geq 0$ :

$$
V_{\infty}-V(t) \geq \gamma \mathrm{e}^{-C_{-} t}+\gamma^{2} \frac{A_{-}}{t^{d+1}} \chi(\{t \geq \bar{t}\})
$$

where $\chi(\{\ldots\})$ is the characteristic function of the set $\{\ldots\}$.

We now compare this result with the analogous one obtained for elastic boundary conditions in [3], in which we have the faster decay $t^{-d-2}$. The physical reason of this different behavior is due to the fact that in presence of stochastic boundary conditions the light particles are emitted with a Maxwellian distribution centered around the body velocity. Therefore a large fraction of the emitted particles have a velocity close to that of the body, and this makes recollisions more likely.

We observe that the results of the present paper, as in the case with elastic collisions, can be extended to two further cases: $V_{0}>V_{\infty}$ and/or a body with a generic convex shape (see [5] for the case of reflecting boundary conditions). 


\section{Proof of Theorem 2.1}

In the sequel we will denote by $C$ any positive constant, possibly depending on $\rho, \beta, R, L, V_{\infty}$, but not on $\gamma$, which is our small parameter.

For any $\gamma \in\left(0, \gamma_{0}\right)$ with $\gamma_{0}$ sufficiently small, we introduce an a.e. differentiable function with bounded derivative, $t \rightarrow W(t)$, such that $W(0)=V_{0}, \lim _{t \rightarrow \infty} W(t)=V_{\infty}$, and satisfying the following properties:

(i) $W$ is increasing over the interval $\left[0, t_{0}\right]$, with

$$
t_{0}=\frac{1}{2 C_{-}} \log \frac{C_{+}}{\gamma}
$$

(ii) There exist two positive constants $A_{+}, A_{-}$such that, for any $t \geq 0$, it is:

$$
V_{\infty}-W(t) \leq \gamma \mathrm{e}^{-C_{+} t}+\gamma^{2} \frac{A_{+}}{(1+t)^{4}}
$$

and, with $\bar{t}=2 t_{0}$,

$$
V_{\infty}-W(t) \geq \gamma \mathrm{e}^{-C_{-} t}+\gamma^{2} \frac{A_{-}}{t^{4}} \chi(\{t \geq \bar{t}\}) .
$$

The two constants $A_{+}$and $A_{-}$, independent of each other and also of $\gamma$ and $\gamma_{0}$, will be fixed later on.

We collect in the following lemma (proved in [3]) some properties of the function $W$ which we will use in the sequel. For $0 \leq s<t$, we set

$$
\langle W\rangle_{s, t}=\frac{1}{t-s} \int_{s}^{t} W(\tau) \mathrm{d} \tau
$$

and

$$
\langle W\rangle_{0, t}=\langle W\rangle_{t} .
$$

Lemma 3.1. Suppose $\gamma_{0}$ sufficiently small. Then:

(i) For any $t>0$ we have:

$$
W(t)>\langle W\rangle_{t}
$$

(ii) $t \rightarrow\langle W\rangle_{t}$ is an increasing function.

(iii) For any $s \in(0, t)$,

$$
\left.\langle W\rangle_{s, t}\right\rangle\langle W\rangle_{t} .
$$

(iv) For any $t>0$, the following bound holds:

$$
W(t)-\langle W\rangle_{t} \leq \frac{C}{1+t}\left(\gamma+A_{+} \gamma^{2}\right) .
$$

The idea of the proof of Theorem 2.1 is the following. We assign the velocity $W$ of the body with the properties just stated. We solve the free Vlasov equation outside a body moving with velocity $W$ and compute the terms $r_{W}^{+}$and $r_{W}^{-}$defined below. Then we solve equation (2.10) for the body with assigned $r_{W}^{+}$and $r_{W}^{-}$, finding a new velocity $V_{W}$. The solution of our problem is the fixed point of the map $W \rightarrow V_{W}$, thus we have to prove for $V_{W}$ the same properties established above for $W$.

Let $X(t)=\int_{0}^{t} W(\tau) \mathrm{d} \tau$ be the position of the center of mass of the body at time $t$. Consider the modified problem:

$$
\frac{\mathrm{d}}{\mathrm{d} t}\left(V_{\infty}-V_{W}(t)\right)=-K(t)\left(V_{\infty}-V_{W}(t)\right)+r_{W}^{+}(t)+r_{W}^{-}(t)
$$

where $K(t)$ is the function introduced in (2.16) with $W(t)$ in place of $V(t)$. Moreover

$$
r_{W}^{+}(t)=\int_{\partial \Omega_{t}^{+}} \mathrm{d} x \int_{v_{x} \leq W(t)} \mathrm{d} v d\left(v_{x}, W(t)\right)\left[f_{-}(x, v ; t)-\rho\left(\frac{\beta}{\pi}\right)^{3 / 2} \mathrm{e}^{-\beta v^{2}}\right]
$$


and

$$
r_{W}^{-}(t)=\int_{\partial \Omega_{t}^{-}} \mathrm{d} x \int_{v_{x} \geq W(t)} \mathrm{d} v d\left(v_{x}, W(t)\right)\left[\rho\left(\frac{\beta}{\pi}\right)^{3 / 2} \mathrm{e}^{-\beta v^{2}}-f_{-}(x, v ; t)\right] .
$$

We want to show that $V_{W}$ behaves like $W$. To this aim we have to estimate $r_{W}^{ \pm}$.

To have a recollision (from the right or from the left basis of the cylinder) it is necessary that $x \in \partial \Omega_{t}^{ \pm}$, and it has to exist a time $s<t$ such that

$$
v_{x}(t-s)=X(t)-X(s)=\int_{s}^{t} W(\tau) \mathrm{d} \tau
$$

that is $v_{x}=\langle W\rangle_{s, t}$ for some $s \in(0, t)$ and

$$
\left|v_{\perp}\right|(t-s) \leq 2 R
$$

Thus by Lemma 3.1 (iii), for a recollision to happen it is necessary that

$$
v_{x} \geq\langle W\rangle_{t} \quad \text { and } \quad\left|v_{\perp}\right| \leq \frac{2 R}{t-s} .
$$

Let us start by estimating $r_{W}^{+}(t)$, proving the following lemma:

Lemma 3.2. For any $t \geq 0$, and $\gamma$ sufficiently small,

$$
C \frac{\gamma^{2}}{(1+t)^{4}} \chi\left(\left\{t \geq t_{0}\right\}\right) \leq r_{W}^{+}(t) \leq C \frac{\left(\gamma+A_{+} \gamma^{2}\right)^{2}}{(1+t)^{4}} .
$$

Proof. Let $\tau(x, v, t) \in(0, t)$ be the first backward recollision time for a particle with velocity $v$, which is hitting the body at $x \in \partial \Omega_{t}^{+}$at time $t$. Omitting the dependence of $\tau$ on $x, v, t$, for notational simplicity, from (2.3) and (2.4) we have, for $x \in \partial \Omega_{t}^{+}$,

$$
\begin{aligned}
f_{-}(x, v ; t)= & \alpha J(\xi, \tau) \mathrm{e}^{-\beta\left(v_{x}-W(\tau)\right)^{2}} \mathrm{e}^{-\beta v_{\perp}^{2}} \chi\left(\left\{\langle W\rangle_{t} \leq v_{x} \leq W(t)\right\}\right) \\
& +\rho\left(\frac{\beta}{\pi}\right)^{3 / 2} \mathrm{e}^{-\beta v^{2}} \chi\left(\left\{-\infty<v_{x}<\langle W\rangle_{t}\right\}\right),
\end{aligned}
$$

where $\xi \in \partial \Omega_{\tau}^{+}$is the collision point at time $\tau$. In fact for $\langle W\rangle_{t} \leq v_{x} \leq W(t)$ the gas particle had a collision with the body at some time instant $\tau<t$, and so it was emitted by the body according to the distribution (2.4), while for $-\infty \leq v_{x} \leq\langle W\rangle_{t}$ the gas particle had no collisions with the body in the past, hence we have to use for it the initial distribution (2.3). Moreover from (2.6) and (3.16) we obtain

$$
\begin{aligned}
J(x, t)=\alpha \int \mathrm{d} v(W(t)- & \left.v_{x}\right) J(\xi, \tau) \mathrm{e}^{-\beta\left(v_{x}-W(\tau)\right)^{2}} \mathrm{e}^{-\beta v_{\perp}^{2}} \\
& \times \chi\left(\left\{\langle W\rangle_{t} \leq v_{x} \leq W(t)\right\}\right) \\
+\int \mathrm{d} v(W(t)- & \left.v_{x}\right) \rho\left(\frac{\beta}{\pi}\right)^{3 / 2} \mathrm{e}^{-\beta v^{2}} \\
& \times \chi\left(\left\{-\infty<v_{x}<\langle W\rangle_{t}\right\}\right) .
\end{aligned}
$$

By Lemma $3.1(i v)$, we have

hence

$$
J(x, t) \leq C \gamma^{2} \sup _{\xi \in \mathbb{R}^{3} \tau \geq 0} J(\xi, \tau)+C,
$$

$$
\sup _{\xi \in \mathbb{R}^{3} \tau \geq 0} \sup _{\tau} J(\xi, \tau) \leq \frac{C}{1-C \gamma^{2}}<C,
$$


for $\gamma$ sufficiently small.

Let us establish first the upper bound for $r_{W}^{+}(t)$. From (3.10), (3.14), (3.16) and (3.19) it follows:

$$
r_{W}^{+}(t) \leq C \int \mathrm{d} v_{\perp} \mathrm{e}^{-\beta v_{\perp}^{2}} \chi\left(\left\{\left|v_{\perp}\right| \leq \frac{2 R}{t-\tau}\right\}\right) \int_{\langle W\rangle_{t}}^{W(t)} \mathrm{d} v_{x} d\left(v_{x}, W(t)\right) .
$$

For $v_{x}$ such that $\tau<t / 2$, we have:

$$
\int \mathrm{d} v_{\perp} \mathrm{e}^{-\beta v_{\perp}^{2}} \chi\left(\left\{\left|v_{\perp}\right| \leq \frac{2 R}{t-\tau}\right\}\right) \leq \frac{C}{(1+t)^{2}}
$$

and we have a first contribution to the upper bound of $r_{W}^{+}(t)$ which is

$$
\frac{C}{(1+t)^{2}} \int_{\langle W\rangle_{t}}^{W(t)} \mathrm{d} v_{x} d\left(v_{x}, W(t)\right) \leq \frac{C}{(1+t)^{2}}\left(W(t)-\langle W\rangle_{t}\right)^{2} .
$$

If $\tau \geq t / 2$, from (3.12) and (3.2) it follows:

$$
\begin{aligned}
v_{x} & =W(t)-\frac{1}{t-\tau} \int_{\tau}^{t} \mathrm{~d} s(W(t)-W(s)) \\
& \geq W(t)-\frac{1}{t-\tau} \int_{\tau}^{t} \mathrm{~d} s\left(V_{\infty}-W(s)\right) \\
& \geq W(t)-\frac{1}{t-\tau} \int_{\tau}^{t} \mathrm{~d} s\left[\gamma \mathrm{e}^{-C_{+} s}+\frac{A_{+} \gamma^{2}}{(1+s)^{4}}\right] \\
& \geq W(t)-\left[\gamma \frac{\mathrm{e}^{-C_{+} \tau}-\mathrm{e}^{-C_{+} t}}{C_{+}(t-\tau)}+C \frac{A_{+} \gamma^{2}}{(1+t)^{4}}\right] .
\end{aligned}
$$

Since

it follows that

$$
\frac{1-\mathrm{e}^{-C_{+}(t-\tau)}}{C_{+}(t-\tau)}<C
$$

$$
v_{x} \geq W(t)-C\left[\gamma \mathrm{e}^{-C_{+} \frac{t}{2}}+\frac{A_{+} \gamma^{2}}{(1+t)^{4}}\right] \geq W(t)-C \frac{\left(\gamma+A_{+} \gamma^{2}\right)}{(1+t)^{4}} .
$$

Hence the second contribution to the upper bound of $r_{W}^{+}(t)$ is

$$
\begin{aligned}
& C \int \mathrm{d} v_{x} d\left(v_{x}, W(t)\right) \chi\left(\left\{W(t)-C \frac{\left(\gamma+A_{+} \gamma^{2}\right)}{(1+t)^{4}} \leq v_{x} \leq W(t)\right\}\right) \\
& \leq C\left(\frac{\left(\gamma+A_{+} \gamma^{2}\right)}{(1+t)^{4}}\right)^{2} .
\end{aligned}
$$

Collecting (3.22), (3.25) and using Lemma 3.1 (iv), we achieve the upper bound in (3.15).

We give now the lower bound of $r_{W}^{+}(t)$. By (3.10), (3.14) and (3.16), we have

$$
\begin{aligned}
& r_{W}^{+}(t)=\int_{\partial \Omega_{t}^{+}} \mathrm{d} x \int \mathrm{d} v \chi\left(\left\{\langle W\rangle_{t} \leq v_{x} \leq W(t)\right\}\right) d\left(v_{x}, W(t)\right) \\
& \times\left[\alpha J(\xi, \tau) \mathrm{e}^{-\beta\left(v_{x}-W(\tau)\right)^{2}} \mathrm{e}^{-\beta v_{\perp}^{2}}-\rho\left(\frac{\beta}{\pi}\right)^{\frac{3}{2}} \mathrm{e}^{-\beta v^{2}}\right] .
\end{aligned}
$$


Moreover, by (3.17),

$$
\begin{aligned}
J(\xi, \tau) & \geq C \int \mathrm{d} v\left(W(\tau)-v_{x}\right) \rho\left(\frac{\beta}{\pi}\right)^{\frac{3}{2}} \mathrm{e}^{-\beta v^{2}} \chi\left(\left\{-\infty<v_{x}<\langle W\rangle_{\tau}\right\}\right) \\
& \geq C
\end{aligned}
$$

Therefore

$$
\begin{aligned}
r_{W}^{+}(t) \geq C \int \mathrm{d} v_{\perp} \mathrm{e}^{-\beta v_{\perp}^{2}} \chi\left(\left\{\left|v_{\perp}\right| \leq \frac{2 R}{t-\tau}\right\}\right) \int_{\langle W\rangle_{t}}^{W(t)} \mathrm{d} v_{x} d\left(v_{x}, W(t)\right) \\
\quad \times\left[C \mathrm{e}^{-\beta\left(v_{x}-W(\tau)\right)^{2}}-\rho\left(\frac{\beta}{\pi}\right)^{\frac{3}{2}} \mathrm{e}^{-\beta v_{x}^{2}}\right] \\
\geq \frac{C}{(1+t)^{2}}\left(W(t)-\langle W\rangle_{t}\right)^{2}
\end{aligned}
$$

since, by the properties of $W$, there exists a positive constant $C_{0}$ (independent of $\gamma$ ) such that, for $V_{0}>C_{0}$ and $v_{x} \in\left[\langle W\rangle_{t}, W(t)\right]$, the term in square brackets in (3.28) is bounded from below by a positive constant. Furthermore, by (3.2) and (3.3),

$$
\begin{aligned}
W(t)-\langle W\rangle_{t} & =\frac{1}{t} \int_{0}^{t} \mathrm{~d} s\left[\left(V_{\infty}-W(s)\right)-\left(V_{\infty}-W(t)\right)\right] \\
& \geq \frac{\gamma}{t} \int_{0}^{t} \mathrm{~d} s\left[\mathrm{e}^{-C_{-} s}-\mathrm{e}^{-C_{+} t}\right]-\gamma^{2} \frac{A_{+}}{(1+t)^{4}} \\
& =\gamma\left[\frac{1-\mathrm{e}^{-C_{-} t}}{C_{-} t}-\mathrm{e}^{-C_{+} t}\right]-\gamma^{2} \frac{A_{+}}{(1+t)^{4}}
\end{aligned}
$$

hence, for $\gamma$ sufficiently small and consequently $t_{0}$ sufficiently large, from (3.28) and (3.29) we obtain the lower bound in (3.15).

For $r_{W}^{-}(t)$ we have an upper bound expressed by the following lemma:

Lemma 3.3. For any $t \geq 0$, and $\gamma$ sufficiently small,

$$
\left|r_{W}^{-}(t)\right| \leq C \chi\left(\left\{t \geq t_{0}\right\}\right)\left(\frac{\gamma+A_{+} \gamma^{2}}{(1+t)^{4}}\right)^{2} .
$$

Proof. We notice first that $r_{W}^{-}(t)=0$ as far as $W$ is increasing, and this justifies the characteristic function in equation (3.30). By (3.12) and (3.3) we have

$$
\begin{aligned}
v_{x}=\langle W\rangle_{s, t} & \leq V_{\infty}-\frac{1}{t-s} \int_{s}^{t}\left[\gamma \mathrm{e}^{-C_{-} \tau}+\gamma^{2} \frac{A_{-}}{\tau^{4}} \chi(\{\tau \geq \bar{t}\})\right] \mathrm{d} \tau \\
& \leq V_{\infty} .
\end{aligned}
$$

Thus from (3.11) and by the form of $f_{-}(x, v ; t)$ (which follows from the analogous of (3.16) and (3.19), written for $x \in \partial \Omega_{t}^{-}$) we obtain:

$$
\left|r_{W}^{-}(t)\right| \leq C \int_{W(t)}^{V_{\infty}} \mathrm{d} v_{x} d\left(v_{x}, W(t)\right) \leq C\left(V_{\infty}-W(t)\right)^{2}
$$


By (3.2) we have

$$
r_{W}^{-}(t) \leq C\left(\gamma \mathrm{e}^{-C_{+} t}+\gamma^{2} \frac{A_{+}}{(1+t)^{4}}\right)^{2},
$$

hence (3.30) follows by noticing that $\mathrm{e}^{-C_{+} t} \leq C /(1+t)^{4}$.

We remark that, from (3.15) and (3.30), it follows

$$
r_{W}^{+}(t)+r_{W}^{-}(t) \geq 0
$$

$\forall t \geq 0$, by choosing $t_{0}$ sufficiently large, that is $\gamma$ sufficiently small.

Now we prove that the function $V_{W}(t)$ satisfying equation (3.9) enjoys, for $\gamma$ suitably small, the same properties as the function $W$, with the same constants $A_{+}, A_{-}$. After this the proof of Theorem 2.1 will follow easily.

From now on we proceed as in [3], and we repeat here the argument for the sake of completeness.

Proposition 3.1. Suppose $\gamma$ sufficiently small. Then:

(i) $t \rightarrow V_{W}(t)$ is an a.e. differentiable function with bounded derivative, increasing over the interval $\left[0, t_{0}\right]$ with

$$
t_{0}=\frac{1}{2 C_{-}} \log \frac{C_{+}}{\gamma}
$$

(ii) For any $t \geq 0$ :

$$
V_{\infty}-V_{W}(t)<\gamma \mathrm{e}^{-C_{+} t}+\gamma^{2} \frac{A_{+}}{(1+t)^{4}}
$$

(iii) For any $t>0$, with $\bar{t}=2 t_{0}$ :

$$
V_{\infty}-V_{W}(t)>\gamma \mathrm{e}^{-C_{-} t}+\gamma^{2} \frac{A_{-}}{t^{4}} \chi(\{t \geq \bar{t}\})
$$

Proof. From equation (3.9) and the Duhamel formula we have:

$$
V_{\infty}-V_{W}(t)=\gamma \mathrm{e}^{-\int_{0}^{t} K(\tau) \mathrm{d} \tau}+\int_{0}^{t} \mathrm{~d} s \mathrm{e}^{-\int_{s}^{t} K(\tau) \mathrm{d} \tau}\left(r_{W}^{+}(s)+r_{W}^{-}(s)\right),
$$

and since $r_{W}^{+}(t)$ and $r_{W}^{-}(t)$ are bounded, by (3.38) and (3.9) $V_{W}$ is a.e. differentiable with bounded derivative.

By the properties of $W$ we have that, for $\gamma$ sufficiently small, $V_{0}<W(t)<V_{\infty}$ for any $t>0$. Consequently for the function $K(t)$ introduced in (2.16) (here defined with $W$ in place of $V$ ) we have $C_{+}<K(t)<C_{-}$for $t>0$. Hence by the non-negativity of $\left(r_{W}^{+}(t)+r_{W}^{-}(t)\right)$, from $(3.38)$ we get, for $t>0$,

$$
V_{\infty}-V_{W}(t)>\gamma \mathrm{e}^{-C_{-} t}
$$

By (3.9), (3.39), (3.15) and (3.30) we have

$$
\frac{\mathrm{d}}{\mathrm{d} t}\left(V_{\infty}-V_{W}(t)\right) \leq-C_{+} \gamma \mathrm{e}^{-C_{-} t}+C \frac{\left(\gamma+A_{+} \gamma^{2}\right)^{2}}{(1+t)^{4}}
$$

and this implies

$$
\frac{\mathrm{d}}{\mathrm{d} t}\left(V_{\infty}-V_{W}(t)\right)<0
$$

for $t \in\left[0, t_{0}\right]$ and $\gamma$ sufficiently small, so that $(i)$ is proved. 
From (3.38), (3.15) and (3.30) it follows:

$$
\begin{aligned}
V_{\infty}-V_{W}(t) & \leq \gamma \mathrm{e}^{-C_{+} t}+\int_{0}^{t} \mathrm{~d} s \mathrm{e}^{-C_{+}(t-s)}\left(r_{W}^{+}(s)+r_{W}^{-}(s)\right) \\
& \leq \gamma \mathrm{e}^{-C_{+} t}+C\left(\gamma+A_{+} \gamma^{2}\right)^{2} \int_{0}^{t} \mathrm{~d} s \frac{\mathrm{e}^{-C_{+}(t-s)}}{(1+s)^{4}}
\end{aligned}
$$

Let us evaluate the integral:

Thus

$$
\begin{aligned}
& \int_{0}^{t} \mathrm{~d} s \frac{\mathrm{e}^{C_{+} s}}{(1+s)^{4}}=\int_{0}^{\frac{t}{2}}(\cdot) \mathrm{d} s+\int_{\frac{t}{2}}^{t}(\cdot) \mathrm{d} s \\
& \leq \frac{\mathrm{e}^{C_{+} \frac{t}{2}}-1}{C_{+}}+\frac{2^{4}}{(2+t)^{4}} \frac{\mathrm{e}^{C_{+} t}-\mathrm{e}^{C_{+} \frac{t}{2}}}{C_{+}} .
\end{aligned}
$$

$$
\begin{aligned}
\int_{0}^{t} \mathrm{~d} s \frac{\mathrm{e}^{-C_{+}(t-s)}}{(1+s)^{4}} & \leq \frac{\mathrm{e}^{-C_{+} \frac{t}{2}}-\mathrm{e}^{-C_{+} t}}{C_{+}}+\frac{2^{4}}{(2+t)^{4}} \frac{1-\mathrm{e}^{-C_{+} \frac{t}{2}}}{C_{+}} \\
& \leq \frac{1}{C_{+}}\left(\mathrm{e}^{-C_{+} \frac{t}{2}}+\frac{2^{4}}{(2+t)^{4}}\right) \leq \frac{C}{(1+t)^{4}}
\end{aligned}
$$

To conclude, there exists a constant $\bar{C}$ such that:

$$
V_{\infty}-V_{W}(t) \leq \gamma \mathrm{e}^{-C_{+} t}+\bar{C}\left(\gamma+A_{+} \gamma^{2}\right)^{2} \frac{1}{(1+t)^{4}}
$$

Therefore to obtain $(i i)$ it is sufficient that

$$
\bar{C}\left(\gamma+A_{+} \gamma^{2}\right)^{2}<A_{+} \gamma^{2}
$$

This inequality is satisfied, for instance, by choosing $A_{+}=2 \bar{C}$ (this fixes $A_{+}$) and $\gamma$ consequently small.

It remains to prove (iii). From (3.38), (3.15) and (3.30) we have, for $t \geq \bar{t}$ and $t_{0}$ sufficiently large (that is $\gamma$ sufficiently small):

$$
\begin{aligned}
V_{\infty}-V_{W}(t) & >\gamma \mathrm{e}^{-C_{-} t}+\int_{0}^{t} \mathrm{~d} s \mathrm{e}^{-C_{-}(t-s)}\left(r_{W}^{+}(s)+r_{W}^{-}(s)\right) \\
& >\gamma \mathrm{e}^{-C_{-} t}+C \int_{t_{0}}^{t} \mathrm{~d} s \mathrm{e}^{-C_{-}(t-s)} \frac{\gamma^{2}}{s^{4}}
\end{aligned}
$$

Now we have

$$
\int_{t_{0}}^{t} \mathrm{~d} s \frac{\mathrm{e}^{-C_{-}(t-s)}}{s^{4}} \geq \frac{1-\mathrm{e}^{-C_{-}\left(t-t_{0}\right)}}{C_{-} t^{4}} \geq \frac{1-\mathrm{e}^{-C_{-} t_{0}}}{C_{-} t^{4}} \geq \frac{1}{2 C_{-} t^{4}},
$$

by (3.1), for $\gamma$ sufficiently small, since $t \geq \bar{t}=2 t_{0}$. Hence:

$$
V_{\infty}-V_{W}(t)>\gamma \mathrm{e}^{-C_{-} t}+C \frac{\gamma^{2}}{t^{4}}
$$

Last inequality enables us to choose $A_{-}$, in such a way that (3.37) is satisfied. This can be done in a consistent manner, since the constant $C$ appearing in (3.48) does not depend of $A_{-}$. Actually it depends of $A_{+}$, nevertheless $A_{-}$can be chosen independently of $A_{+}$for $\gamma$ sufficiently small. 
Any solution to this problem satisfies bounds (2.23) and (2.24). Consider in fact a solution $(V, f)$ of the problem. By continuity of $V$ there exists a time interval in which inequalities (2.23)-(2.24) hold strictly. Let $T$ be the first time for which our strict inequalities are violated. The same arguments used in Proposition 3.1 (replacing $W$ by $V$ ) show that (2.23)-(2.24) hold strictly in the time interval $(0, T]$, since in this interval $V$ enjoys the same properties as $W$. Then $T$ must be infinite. This concludes the proof of Theorem 2.1.

By using the results of Section 3 it is easy to prove the existence of the pair $(V, f)$, by studying the fixed point of the map $W \rightarrow V_{W}$ by means of Schauder's theorem (see [4] for details).

\section{REFERENCES}

[1] W. Braun and K. Hepp, The Vlasov dynamics and its fluctuations in the $1 / N$ limit of interacting classical particles. Comm. Math. Phys. 56 (1977) 101-113.

[2] P. Buttà, E. Caglioti and C. Marchioro, On the long time behavior of infinitely extended systems of particles interacting via Kac Potentials. J. Stat. Phys. 108 (2002) 317-339.

[3] S. Caprino, C. Marchioro and M. Pulvirenti, Approach to equilibrium in a microscopic model of friction. Comm. Math. Phys. 264 (2006) 167-189.

[4] S. Caprino, G. Cavallaro and C. Marchioro, On a microscopic model of viscous friction. Math. Models Methods Appl. Sci. 17 (2007) 1369-1403.

[5] G. Cavallaro, On the motion of a convex body interacting with a perfect gas in the mean-field approximation. Rend. Mat. Appl. 27 (2007) 123-145.

[6] R.L. Dobrushin, Vlasov equations. Sov. J. Funct. Anal. 13 (1979) 115-123.

[7] C. Gruber and J. Piasecki, Stationary motion of the adiabatic piston. Physica A 268 (1999) 412-423.

[8] J.L. Lebowitz, J. Piasecki and Y. Sinai, Scaling dynamics of a massive piston in a ideal gas, in Hard Ball Systems and the Lorentz Gas, Encycl. Math. Sci. 101, Springer, Berlin (2000) 217-227.

[9] H. Neunzert, An Introduction to the Nonlinear Boltzmann-Vlasov Equation, in Kinetic Theories and the Boltzmann Equation, Montecatini (1981), Lecture Notes in Math. 1048, Springer, Berlin (1984) 60-110.

[10] H. Spohn, On the Vlasov hierarchy. Math. Meth. Appl. Sci. 3 (1981) 445-455. 